\title{
Adaptive Segmentation of Multi-modal 3D Data Using Robust Level Set Techniques
}

\author{
Aly Farag and Hossam Hassan \\ Computer Vision and Image Processing Laboratory \\ University of Louisville, Louisville, KY 40292. \\ \{hossam,farag\}@cvip.uofl.edu \\ http://www.cvip.louisville.edu
}

\begin{abstract}
A new 3D segmentation method based on the level set technique is proposed. The main contribution is a robust evolutionary model which requires no fine tuning of parameters. A closed 3D surface propagates from an initial position towards the desired region boundaries through an iterative evolution of a specific 4D implicit function. Information about the regions is involved by estimating, at each iteration, parameters of probability density functions. The method can be applied to different kinds of data, e.g for segmenting anatomical structures in $3 \mathrm{D}$ magnetic resonance images and angiography. Experimental results of these two types of data are discussed.
\end{abstract}

\section{Introduction}

Both surgical planning and navigation benefit from image segmentation. Also the $3 \mathrm{D}$ segmentation of anatomical structures is very important for medical visualization and diagnostics. The segmentation process is still a challenging problem because of image noise and inhomogeneities. Therefore this process can not depend only on image information but also has to exploit the prior knowledge of shapes and other properties of the structures to be segmented.

In many cases, the 3D segmentation is performed using deformable models. The mathematical foundation of such models represents the confluence of physics and geometry [1. The latter represents an object shape and the former puts constraints on how the shape may vary over space and time. Deformable models have had great successes in imaging and computer graphics. In particular in [2], the deformable models recover the object's structure using some properties of its shape. The model evolves iteratively towards the steady state of energy minimization. But the disadvantage of this method is that the initial contour should be close to the final one. The model faces also problems with topological changes of a complex structure.

Level set techniques of segmentation overcome problems of the classical deformable models 345. A curve in 2D or a surface in 3D evolves in such a way as to cover a complex shape or structure. Its initialization is either manual or automatic and it need not to be close to the desired solution. But these methods depend on a big number of parameters to be tuned for the success of the process. 
In 6], a more efficient 3D segmentation technique was proposed. In this approach, surface evolution is controlled by current probabilistic region information. Probability density functions for the object and the background are estimated using the Stochastic Expectation Maximization algorithm (SEM). The level set model designed is based on these density functions. But this method can work only for bimodal images, and this may be too restrictive for many applications.

Also in [78], new segmentation methods were proposed using level set techniques. The former provided results for segmenting thin structure while the latter gave results for some real and synthetic images.

In this paper, a novel and robust segmentation based on the level set technique is proposed. A statistical model of regions is explicitly embedded into partial differential equations describing the evolution of the level sets. The probability density function for each region is modelled by a Gaussian with adaptive parameters. These parameters and the prior probability of each region are automatically re-estimated at each iteration of the process. The level set model designed depends on these density functions. The region information over the image is also taken into account.

Initialization of level set functions is very important for success of this segmentation process. An automatic seed initialization is used to accelerate the process and make it less sensitive to noise. The chosen initialization needs an accurate estimate of the parameters for each class. The SEM algorithm is used to give initial estimates of class parameters. During the level sets evolution, these parameters are iteratively re-estimated in order to obtain more accurate segmentation. Our work differs from that in 6] due to its suitability for multi-modal images and due to adaptive estimation of the probability density functions. Our experiments in 3D segmentation of MR images and angiography demonstrate the accuracy of the algorithm.

The paper is organized as follows. Section 2 considers the proposed level set formalism. Section 3 explains in brief the estimation of probability densities of image signals. The proposed evolutionary surface model is presented in Section 4 Experiments with simulated and real 3D images are discussed in Section 5 .

\section{Surface Modelling by Level Sets}

Within the level set formalism [9], the evolving surface is a propagating front embedded as the zero level of a $4 \mathrm{D}$ scalar function $\phi(x, t)$. This hypersurface is usually defined as the signed distance function positive inside, negative outside, and zero on the boundary of a region. The continuous change of $\phi$ can be described by the partial differential equation:

$$
\frac{\partial \phi(x, t)}{\partial t}+F|\nabla \phi(x, t)|=0,
$$

where $F$ is a scalar velocity function depending on the local geometric properties (local curvature) of the front and on the external parameters related to the input 
data e.g, image gradient. The hypersurface $\phi$ deforms iteratively according to $F$, and the position of the 3D front is given at each iteration step by the equation $\phi(x, t)=0$. Practically, instead of Eq. 11 the value $\phi\left(x, t_{n+1}\right)$ at step $n+1$ is computed from $\phi\left(x, t_{n}\right)$ at step $n$ by the relation:

$$
\phi\left(x, t_{n+1}\right)=\phi\left(x, t_{n}\right)-\triangle t \cdot F\left|\nabla \phi\left(x, t_{n}\right)\right|,
$$

The design of the velocity function $F$ plays the major role in the evolutionary process. Among several formulations proposed in [1011], we have chosen the following formulation:

$$
F=\nu-\epsilon k,
$$

where $\nu=1$ or -1 for the contracting or expanding front, respectively, $\epsilon$ is a smoothing coefficient always small with respect to 1 , and $k$ is the local curvature of the front defined in the $3 \mathrm{D}$ case as follows:

$$
\begin{gathered}
k=\left(\left(\phi_{x x}+\phi_{y y}\right) \phi_{z}{ }^{2}+\left(\phi_{x x}+\phi_{z z}\right) \phi_{y}{ }^{2}\right. \\
+\left(\phi_{z z}+\phi_{y y}\right) \phi_{x}{ }^{2}-2 \phi_{x} \phi_{y} \phi_{x y}-2 \phi_{x} \phi_{z} \phi_{x z} \\
\left.-2 \phi_{z} \phi_{y} \phi_{z y}\right) /\left(2\left(\phi_{x}^{2}+\phi_{y}^{2}+\phi_{z}^{2}\right)^{3 / 2}\right),
\end{gathered}
$$

The latter parameter acts as a regularization term.

With this representation a single level set either contracts until vanishing or expands to cover all the space. To stop the evolution at the edge, $F$ can be multiplied by a value which is a function of the image gradient[12]. But if the edge is missed, the surface can not come back. So to depend only on the edge is not sufficient for accurate segmentation and other information from the image should be used.

The segmentation partitions the image into regions each belonging to a certain class. In our approach a separate level set function is defined for each class and automatic seed initialization is used. Given parameters of each class, the volume is initially divided into equal non-overlapped sub-volumes. For each subvolume, the average gray level is used to specify the most probable class with the initial parameters estimated by the SEM. Such initialization differs from that in [13] where only the distance to the class mean is used. Then a signed distance level set function for the associated class is initialized. Therefore selection of the class parameters is very important for the successful segmentation. The probability density functions of classes are embedded into the velocity term of each level set equation. The parameters of each one of these density functions are re-estimated at each iteration. The automatic seed initialization produces initially non-overlapped level set functions. The competition between level sets based on the probability density functions stops the evolution of each level set at the boundary of its class region.

\section{Estimation of Intensity Probability Density Functions}

A segmented image $I$ consists of homogeneous regions characterized by statistical properties related to a visual consistency. The inter-region transitions are 
assumed to be smooth. Let $\Omega \in R^{p}$ be open and bounded $p$-dimensional volume. Let $I: \Omega \rightarrow R$ be the observed $p$-dimensional image data. We assume that the number of classes $K$ is known. Let $p_{i}(I)$ be the intensity probability density function of class $i$. Each density function must represent the region information to discriminate between two different regions. In our experience Gaussian models show satisfactory results in medical image segmentation. In this work we also use such density functions and associate the mean $\mu_{i}$, variance $\sigma_{i}^{2}$, and prior probability $\pi_{i}$ with each class $i$. The priors satisfy the obvious condition:

$$
\sum_{i=1}^{K} \pi_{i}=1
$$

In accord to the estimation method in [14], the model parameters are updated at each iteration as follows:

$$
\begin{gathered}
\mu_{i}=\frac{\int_{\Omega} H_{\alpha}\left(\phi_{i}\right) I(x) d x}{\int_{\Omega} H_{\alpha}\left(\phi_{i}\right) d x} . \\
\sigma_{i}^{2}=\frac{\int_{\Omega} H_{\alpha}\left(\phi_{i}\right)\left(\mu_{i}-I(x)\right)^{2} d x}{\int_{\Omega} H_{\alpha}\left(\phi_{i}\right) d x} .
\end{gathered}
$$

We propose the following equation to estimate the prior probability by counting the number of pixels in each region and divide it by the total number of pixels:

$$
\pi_{i}=\frac{\int_{\Omega} H_{\alpha}\left(\phi_{i}\right) d x}{\sum_{i=1}^{K} \int_{\Omega} H_{\alpha}\left(\phi_{i}\right) d x} .
$$

Here, $H_{\alpha}(z)$ is the Heaviside step function defined in [15] as a smoothed differentiable version of the unit step function. The function $H_{\alpha}(z)$ changes smoothly at the boundary of the region. By the above equations, the model parameters are estimated based on the region information.

\section{Evolutionary Surface Model}

The term $(\nu= \pm 1)$ in Eq. 3 specifies the direction of the front propagation. Several approaches were developed to make all fronts either contracting or expanding (see, e.g., [16]) in order to evolve in both directions and avoid overlaps between the regions. The problem can be reformulated as classification of each point at the evolving front. If the point belongs to the associated class, the front expands otherwise it contracts.

\subsection{PDE System}

The classification decision is based on Bayes' decision [17] at point $x$ as follows:

$$
i^{*}(x)=\arg \max _{i=1, . ., K}\left(\pi_{i} p_{i}(I(x))\right) .
$$


The term $(\nu)$ for each point $x$ is replaced by the function $\nu_{i}(x)$ so the velocity function is defined as:

$$
F_{i}(x)=\nu_{i}(x)-\epsilon \cdot k(x), \forall i=1 . . K .
$$

where

$$
\nu_{i}(x)= \begin{cases}-1 & \text { if } i=i^{*}(x) \\ 1 & \text { otherwise }\end{cases}
$$

If the pixel $x$ belongs to the front of the class $i=i^{*}(x)$ associated to the level set function, the front will expand, otherwise it will contract. Now, we put the Eq. 1 in the general form using the derivative of the Heaviside step function $\left(\delta_{\alpha}(z)\right)$ [13] as follows :

$$
\frac{\partial \phi_{i}(x, t)}{\partial t}=\delta_{\alpha}\left(\phi_{i}(x, t)\right)\left(\epsilon \cdot k(x)-\nu_{i}(x)\right)\left|\nabla \phi_{i}(x)\right| .
$$

The function $\delta_{\alpha}(z)$ selects the narrow band points around the front. Solution of the PDEs requires numerical processing at each point of the image or volume which is a time consuming process. Actually we are interested only in the changes of the front, so that the solution is important at the points near the front. Such narrow band points are selected in Eq. 12 Points outside the narrow band are given large positive or large negative values to be excluded from processing in order to accelerate the iterations.

\section{Experimental Results}

\subsection{Brain MR Images}

We have four classes: ( $i$ )bones, (ii)gray matter (GM), (iii) white matter (WM), and $(i v)$ cerebral spinal fluid (CSF). Applying the automatic seed initialization directly may result in miss-classifying some pixels that share the gray level range of the brain as shown in Fig. 1. That may lead to segment the eye as a brain for example. Therefore gray levels only are not sufficient for accurate segmentation. To solve this problem, the level sets for the classes have the automatic seed initialization except the (GM) class is initialized manually inside the volume as small balloons. But such initialization yields a lower prior probability of the (GM) growing region than it should have in Eq. 8 comparing to the other two classes. To avoid this problem, we compute the prior probabilities for all the classes by Eq. 8 but for the (GM) prior use the condition of Eq. 5: $\pi_{2}=1-\pi_{1}-$ $\pi_{3}-\pi_{4}$ and Eq. 8 is modified as follows:

$$
\pi_{i}=\frac{\int_{\Omega} H_{\alpha}\left(\phi_{i}\right) d x}{\int_{\Omega} d x}, \forall i=1,3,4 .
$$

After these modifications, the initial balloons (Fig. 1 - 1-right image) will evolve to cover the GM region without overlapping other regions. We only show results for WM/GM regions that represent the brain. 
Table 1. Classification accuracy at different noise and inhomogeneity levels.

\begin{tabular}{|c|c|c|c|c|}
\hline Noise and Inhomogeneity & Bones & GM & WM & CSF \\
\hline $0 \%-0 \%$ & $99.8 \%$ & $98.1 \%$ & $99.1 \%$ & $98.5 \%$ \\
$3 \%-20 \%$ & $97.9 \%$ & $97.5 \%$ & $98.3 \%$ & $97.2 \%$ \\
$9 \%-40 \%$ & $97.3 \%$ & $97.2 \%$ & $96.4 \%$ & $95.4 \%$ \\
\hline
\end{tabular}
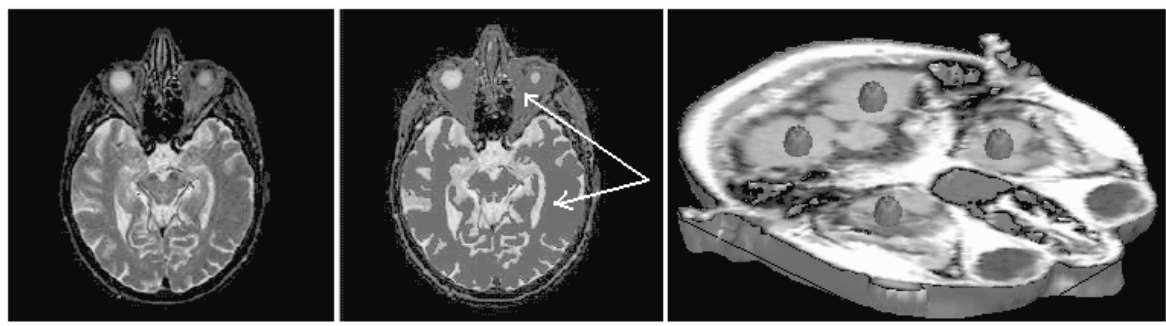

Fig. 1. T2 weighted MR image (left), showing that some areas outside the brain may have the same range of gray levels of the GM(the areas marked by the arrows) (middle), and the initialization of level set function of the GM inside the volume (right).
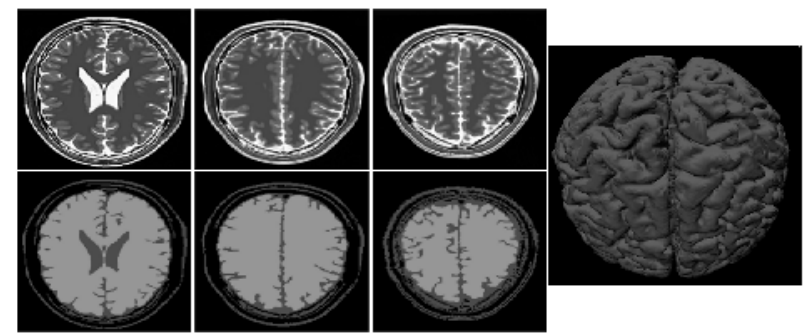

Fig. 2. Brain segmentation (light gray areas in the bottom images) and its $3 \mathrm{D}$ view to the right.

Simulated T2-weighted MR 3D image data sets of the size $181 \times 217 \times 174$ are downloaded from the web sight http://www.bic.mni.mcgill.ca/brainweb/. These data sets have different noise and inhomogeneity levels. Table 5.1 shows the accuracy of the approach for each region. Figure 2 shows results of the segmented brain and its 3D view.

\subsection{Magnetic Resonance Angiography}

Magnetic Resonance Angiography(MRA) is based on amplification of signals from blood vessels and suppression of signals from other tissues. The blood vessels appear as lighter spots in the image. Traditional segmentation needs an extra post-processing to remove the non-blood-vessel areas from the final region maps 18,19. In our approach, the data set has three classes, namely, CSF with bones, GM with WM, and blood vessels (BV) combined with the fat around 


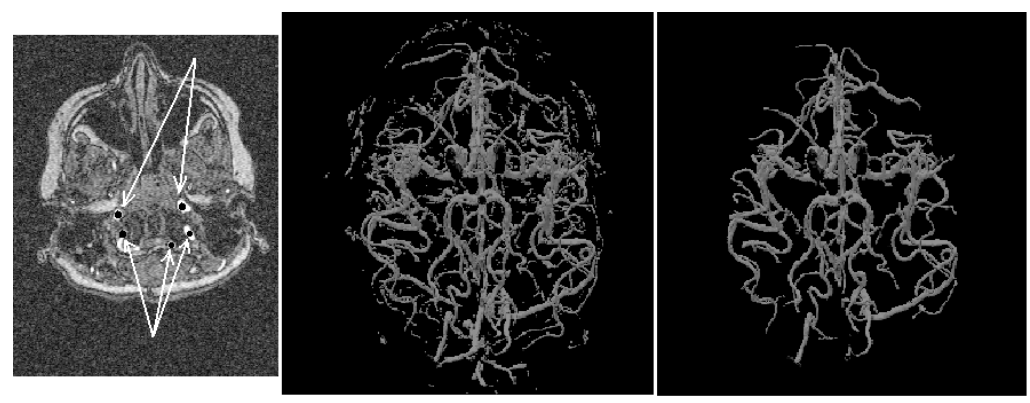

Fig. 3. MRA slice with the cross-sections of the initialized BV level set functions (black circles) (left), visualization of the segmented $512 \times 512 \times 93$ data set using traditional techniques in [18] with fat(middle), and the visualization of our results(right).

the brain which has practically the same range of the gray levels as the blood vessels. Once again, the level set function for the BV class is initialized manually as balloons inside the vessels that have the largest cross sectional area as shown in Fig. 3-left image, and the prior probabilities are estimated as in previous section (with the obvious changes of the class indices). As a result, the fat does not appear in the final segmentation results. Data sets were collected using a Picker 1.5T Edge MRI scanner. It consists of 512x512x93 axial slices with slice thickness $1 \mathrm{~mm}, \mathrm{TR}=27 \mathrm{~ms}, \mathrm{TE}=6 \mathrm{~ms}$. The proposed segmentation approach is tested on 20 data sets.

\section{Conclusions and Future Research}

We developed a simple and fast statistical evolutionary model based on the level set techniques. The model does not need fine tuning of weighting parameters, but the number of classes (regions) has to be known. Each class is assigned with a level set function, and the SEM algorithm provides initial estimates of parameters of the Gaussian model of each class. These estimates permit us to initialize the level sets near to the optimal solution in order to reduce considerably the number of iterations. The Gaussian models of each class are re-estimated at each iteration.

Experiments with real and simulated 3D images confirm that the proposed method is robust and accurate. We expect it may fail on the images where simple Gaussian models cannot discriminate between the regions which makes a limitation. In future work we are going to use more general parametric types of probability distributions which can be estimated with the level sets evolution. 


\section{References}

1. S. Osher and N. Paragios, Geometric Level Set Methods in Imaging, Vision, and Graphics, Springer, 2003.

2. D. Terzopoulos, "Regularization of inverse visual problems involving discontinuities," IEEE Tr. on PAMI, 8(2):413-424, 1986.

3. V. Caselles, R. Kimmel, and G. Sapiro., "Geodesic active contours," IJCV, 22:6179, 1997.

4. H-K. Zaho, T. Chan, B. Merriman, and S. Osher, "A variational level set approach to multiphase motion," J. of Computational Physics, 127:179-195, 1996.

5. R. Goldenberg, R. Kimmel, E. Rivlin, and M. Rudzsky, "Cortex Segmentation: A Fast Variational Geometric Approach," IEEE Tr. on Medical Imaging, Vol. 21, No. 2, pp. 1544-1551, December 2002.

6. C. Baillard, and C. Barillot, "Robust 3D segmentation of anatomical structures with level sets," MICCAI LNCS 1935, Pages 236-245, Pittsburgh, Pennsylvania, October 2000.

7. M. Holtzman-Gazit, D. Goldsher, and R. Kimmel. "Hierachical Segmentation of Thin Structures in Volumetric Medical Images ," MICCAI, Montreal, Canada, November 16-18, 2003.

8. T.Kadir, and M. Brady. "Unsupervised Non-parametric Region Segmentation Using Level Sets," ICCV, Niece, France, October 11-17, 2003.

9. J.A. Sethian, Level Set Methods and Fast Marching Methods, Combridge University Press, USA, 1999.

10. J. Gomes and O. Faugeras, "Reconciling distance functions and Level-Sets,", Technical Report 3666,INRIA, April 1999.

11. N. Paragios and R. Deriche., "Unifying boundary and region-based information for geodesic active tracking," CVPR, Vol. 2, pp 300-305, Fort Collins, Colorado, jun. 1999.

12. R. Malladi, J. Sethian, and B. Vemuri. "Shape modeling with front propagation: A level set approach," IEEE Tr. on PAMI, 17(2):158-175, febreuary, 1995.

13. C. Samson, L. Blanc-Féraud, G. Aubert, and J. Zerubia. "Multiphase Evolution and Variational Image Classification," Technical Report 3662, INRIA, France, 1999.

14. T. Chan and L. Vese. "A multiphase level set framework for image segmentation using the mumford and shah model.," International Journal of Computer Vision, 50(3):271-293,2002.

15. T. Chan, B.Sandberg, and L. Vese." Active Contours without Edges for Vector Valued Images," Journal of Visual Communications and Image Representations, 2:130-141, 2000.

16. X. Zeng, L.H. Staib, R.T. Schultz, H. Tagare, L. Win, and J.S. Duncan. "A new approach to 3D sulcal ribbon finding from MR images," MICCAI, pages 148-157, sep. 1999.

17. R. Duda, P. Hart, and D. Stork. "Pattern Classification ," John Wiley and Sons Inc., 2001.

18. M.Sabry, A. Farag, S. Hushek, and T. Moriarity. "Statistical-Based Approach for Extracting 3D Blood Vessels from TOF-MRA Data," MICCAI, Montreal, Canada, November 16-18, 2003.

19. H. Hassan, A. Farag. "MRA Data Segmentation Using Level Sets," in Proc IEEE International Conference on Image Processing, Barcelona, Spain, Sep. 14-17, 2003. 